\title{
Educating about Nutrition
}

\section{Aradhana Dixit*}

Department of Food and Nutrition, India

*Corresponding Author: Aradhana Dixit, Department of Food and Nutrition, India.

Received: September 30, 2019; Published: October 04, 2019

Dietitians/Nutritionist can actually trained patient about many aspects... These can be done in form of sessions... in dietetic clinics... in hospitals. The patient can be educated about:

- $\quad$ Eating healthier foods: Various tips can be given about eating in healthier way. Like using fats and oils appropriately... avoid too much sodium, taking iodized salts or taking complete or balanced diet.

- Counting plate waste: sometimes diet is prescribed and various nutrients are counted but plate waste is neglected. Patients and caretakers should be taught about.

- Burning calories: Restrictions should be taught if necessary but burning calories through exercise or yoga can be taught by experts.

\section{Good cooking}

- Methods of cooking ...like best to eat raw foods...deep frying gives palatability at the same time may be source of saturated fats.

- Cutting vegetables: Too much chopped vegetables and than washed under tap water may lead to nutrient losses.

- Boiling and draining remaining water: May lead to nutrient losses.

\section{Some tips}

- $\quad$ Eat full fruits rather than sugary juices

- Go nuts

- $\quad$ Go veggies

- Drink plenty water.

These are some tips.. but nutrition sessions can be as creative as they can...
There are lots of challenges to arrange them... some of them can be.

- Taking permission and availability of space

- Convincing patients and having max strength as per req.

- $\quad$ Scheduling counseling

- Occupancy of healthcare professionals.

But if tried.. if these can be overcome!!!

Volume 3 Issue 11 November 2019

(C) All rights are reserved by Aradhana Dixit. 